\title{
Comunicación sexual: diferencias entre padres de hijos con y sin discapacidad
}

\author{
Sexual communication: Differences between parents \\ of disabled and non-disabled children
}

\author{
Susana Xóchitl Bárcena Gaona y Benjamin C. Jenkins ${ }^{2}$
}

\begin{abstract}
RESUMEN
El objetivo de este estudio fue comparar las características sociodemográficas, nivel de conocimientos, creencias, estereotipos, expectativas y comunicación sexual entre padres de hijos con y sin discapacidad. Se trabajó con una muestra no probabilística de 152 padres con hijos adolescentes distribuidos en los dos grupos referidos. De ellos, $77.6 \%$ fueron mujeres cuya edad promedio fue de 45 años. Los resultados más importantes señalan que los padres de hijos con discapacidad tienen más estereotipos y se comunican menos con sus hijos que los padres de hijos sin discapacidad. Los resultados se discuten en términos de las implicaciones en la promoción de la salud sexual de estas personas.
\end{abstract}

Palabras clave: Adolescentes; Discapacidad; Padres; Comunicación; Sexualidad.

\begin{abstract}
The aim of the present study was to compare the sociodemographic characteristics, level of knowledge, beliefs, stereotypes, expectations, and sexual communication between parents of disabled and non-disabled children. Participants consisted of 152 parents with an average age of 54 with teenage children assigned to two groups: parents of disabled children (PDC) and parents of non-disabled children (PNC). Results show that PDCs have more stereotypes and communicate less with their children than PNCS. These findings are discussed in terms of the implications for promoting sexual health of persons with disabilities.
\end{abstract}

Key words: Adolescents; Disability; Parents; Communication; Sexuality.

\section{INTRODUCCIÓN}

$\mathrm{L}$

os jóvenes mexicanos inician su vida sexual a los 15 años en promedio, y más de la mitad lo hace sin protección para prevenir un embarazo no deseado o una infección de transmisión sexual (ITS), incluido el VIH/sida (Instituto Nacional de Estadística y Geografía [INEGI] y Consejo Nacional de Población [CONAPO], 2015). Se estima que, anualmente, 20\% de los embarazos corresponde a mujeres menores de 19 años (INEGI, 2010). A pesar de que el índice general de natalidad disminuye cada año, el de madres adolescentes observa una tendencia inversa; asimismo, se calcula que $41.5 \%$ de los casos de VIH/sida diagnosticados corresponden a jóvenes de entre 15 y 29 años de edad (Centro Nacional para la Prevención y el Control del VIH y el Sida [CENSIDA], 2014).

\footnotetext{
${ }^{1}$ Facultad de Estudios Superiores Iztacala, Universidad Nacional Autónoma de México. Av. de Los Barrios No. 1, Los Reyes Iztacala, 54090 Tlalnepantla, Edo. de México, México, tel. (55)56-23-13-33, ext. 39753, correo electrónico: s.barcena@campus.iztacala.unam.mx. Artículo recibido el 18 de octubre de 2016 y aceptado el 8 de mayo de 2017.

${ }^{2}$ Western New Mexico University, P.O. Box 680, 88062 Silver City, New Mexico. USA, correo electrónico: benjamin.jenkins@wnmu.edu.
} 
La falta de información precisa y oportuna sobre el uso de preservativos y otros métodos anticonceptivos contribuye significativamente a engrosar el número de los problemas de salud sexual referidos anteriormente. En el caso de los jóvenes con discapacidad, la situación es más grave debido a que sufren discriminación y es limitado su acceso a los servicios educativos y de salud (Secretaría de Educación Pública, 2016; Organización Mundial de la Salud [OMS], 2016). Además, se cree erróneamente que son asexuales o "ángeles eternos", por lo que no están en riesgo de contraer ITS, y se desestima asimismo que estén expuestos a abusos sexuales; de hecho, no se les brinda educación sexual por considerarla innecesaria (Rivera, 2008).

En México no existen datos oficiales acerca de la salud sexual de personas con discapacidad; no obstante, en la Encuesta Mundial sobre VIH/sida y Discapacidad, efectuada por la Universidad de Yale para el Banco Mundial (cfr. World Bank, 2009), se indica que las personas con discapacidad mantienen una vida sexual activa y que están más expuestas a diversos factores de riesgo. Datos del Departamento de Justicia de Estados Unidos (cfr. Harrell, 2015) señalan que la tasa de violación y asalto sexual es dos veces más alta en personas con discapacidad: 22 por cada 1,000, que en personas sin discapacidad: 10 por cada 1,000. Estos datos confirman que privar a los jóvenes de recibir educación sexual es colocarlos en una situación de riesgo.

Muchos estudios muestran consistentemente que los jóvenes que reciben educación sexual tienden a retardar la edad de su debut sexual y adoptan conductas protectoras al inicio de su vida sexual (Schaalma, Abraham, Gilmore y Kok, 2004); sin embargo, en el caso de las personas con discapacidad eso supone una doble desventaja, pues los programas orientados a la promoción de la salud y la prevención no están diseñados para ellos (OMS, 2015), y las experiencias y situaciones que les ayuden a comprender su propia sexualidad se encuentran restringidas (Navarro y Hernández, 2012).

Por ende, los padres desempeñan un papel primordial en la promoción de conductas sexuales saludables de sus hijos a través de la comunicación sobre la sexualidad que establecen con ellos (Bárcena, Robles y Díaz-Loving, 2013). Está de- mostrado que los jóvenes que conversan con sus padres sobre temas de sexualidad retardan la edad de debut sexual (Markham, Lormand y Gloppen, 2010), usan condón en su primera relación sexual (Frías, Barroso, Rodríguez, Moreno y Robles, 2008) y tienen un desarrollo sexual más positivo (Dilorio, Pluhar y Belcher, 2003). Por el contrario, cuando los padres no hablan con sus hijos acerca de su sexualidad dan pie a que adquieran información insuficiente y desvirtuada de su grupo de pares, la televisión y la Internet (Caricote, 2008).

En los hogares de estas personas rara vez se aborda la sexualidad (Gutiérrez, 2010; Robles, Guevara, Pérez y Hermosillo, 2013). A menudo, los padres experimentan temor ante la posibilidad de que sus hijos tengan una vida sexual, y piensan que al alejarlos de todo aquello que haga referencia a la sexualidad harán que esta no surja; suelen asimismo adoptar conductas sobreprotectoras hacia sus hijos, lo que limita la autonomía y reduce las actividades de los mismos al ámbito familiar y a la institución que les brinda servicios (López, 2006; Navarro y Hernández, 2012). Además, los padres pueden considerar que cualquier expresión de la sexualidad de sus hijos es inaceptable, y como resultado de esto no están dispuestos a discutirla (Morales, López y Mullet, 2011).

Investigaciones recientes en padres de hijos con discapacidad indican que aquellos son más propensos a comunicarse sobre sexualidad con estos cuando tienen una percepción positiva y realista sobre la discapacidad y la sexualidad (Ballan, 2012). En el mismo sentido, los padres más jóvenes tienden a aceptar más positivamente el desarrollo sexual de sus hijos y, por consiguiente, se comunican más frecuentemente con ellos sobre dichos temas (Cuskelly y Bryde, 2004; Karellou, 2007). Cabe destacar que la comunicación se establece más fácilmente cuando el padre es del mismo sexo que el hijo (Jerman y Constatine, 2010). Al igual que en los jóvenes sin discapacidad, los discapacitados recurren también a su grupo de amigos y a los medios electrónicos en busca de información sobre la sexualidad cuando sus padres no se las brindan (Pownall, Jahoda, Hastings y Kerr, 2011).

En la población de padres que tienen hijos sin discapacidad se observa que sus características influyen en el diálogo que establecen con ellos acerca de la sexualidad. Los padres más jóvenes 
(Einsenberg, Sievening, Bearinger, Swain y Resnick, 2005) y con un nivel escolar superior (Gallegos, Villaruel, Gómez, Onofre y Zhou, 2007) se involucran más en dicha comunicación, lo que ocurre más con las madres que con los padres (Meshke, Bartolomé y Zentael, 2002). También el grado de conocimientos sobre el sida, el sexo seguro, los estereotipos de género y las creencias influyen en la comunicación (Lehr, Demi, Dilorio y Facteau, 2005); por ejemplo, los padres con más estereotipos de género enseñan a sus hijos que la manera en que se experimenta el romance y las relaciones sexuales deben ser distintas para hombres y mujeres y que a estos se les concede una mayor libertad (Shearer, Hosterman, Gillen y Lefkowitz, 2005). Asimismo, los padres con creencias negativas hacia el uso del condón enseñan a sus hijos que la mejor forma de cuidar su salud es abstenerse de tener relaciones, mientras que los padres con creencias más positivas les enseñan a prevenir los riesgos sexuales mediante el condón (Lefkowitz y Stoppa, 2006).

Pese a que los estudios referidos anteriormente se llevaron a cabo con padres de hijos sin discapacidad, es posible suponer que las variables analizadas en dicha población sean también importantes para los padres que tienen hijos con discapacidad. Sin embargo, es posible suponer que la frecuencia y magnitud de cada una de aquellas sean distintas. Por ejemplo, no se preocupan por buscar información sexual bajo el supuesto de que sus hijos no requieren esa información, no consideran importante hablar sobre ello y, en consecuencia, no dialogan con sus hijos.

Por lo anterior, el objetivo de esta investigación fue comparar las características sociodemográficas, el grado de conocimientos, las creencias y estereotipos, las expectativas hacia la comunicación y la frecuencia de la misma sobre la sexualidad en ambos grupos.

\section{MÉTODO}

\section{Participantes}

Se empleó un muestreo no probabílistico intencional por cuotas mediante el cual se seleccionaron 152 padres con hijos adolescentes, distribuidos en dos grupos: padres de hijos con discapacidad (PCD) y padres de hijos sin discapacidad (PSD). En el caso del primer grupo, se acudió a asociaciones formales e informales del Estado de México (México) que brindan atención a personas con discapacidad, y se pidió la colaboración de sus directores para encuestar a los padres, cuyos hijos tuvieran entre 12 y 19 años y presentaran algún tipo de discapacidad (intelectual, auditiva, visual o motriz). Respecto al segundo grupo, se solicitó el permiso a los directores de escuelas secundarias de la entidad para aplicar a los padres los instrumentos durante una de la reuniones escolares que se llevan a cabo bimestralmente con motivo de la firma de boletas. La muestra estuvo compuesta principalmente por mujeres $(77.6 \%)$, cuya edad promedio fue de 45 años (D.E. $=8$ ); casadas $(59.9 \%)$ o en unión libre $(13.8 \%)$ y con un nivel educativo bajo (30.5\% con secundaria y $30.5 \%$ con primaria).

\section{Instrumentos}

En este estudio se emplearon cinco instrumentos de la Encuesta Estudiantil sobre Salud Sexual (EESS) elaborada por Bárcena et al. (2013), adaptados y validados por Bárcena (2013) en una muestra de padres de familia, los cuales se describen a continuación:

Características sociodemográficas. Se recabó información sobre el parentesco paterno del participante y se emplearon cinco reactivos para conocer su sexo, edad, estado civil y grado escolar.

Prueba de conocimientos. La prueba estuvo constituida por dieciocho reactivos; doce de ellos destinados a obtener información del grado de conocimientos acerca de las formas de prevención y transmisión del VIH, así como las consecuencias sociales y psicológicas del embarazo adolescente; los seis reactivos restantes correspondieron al uso correcto del condón. Las opciones de respuesta para cada reactivo fueron "cierto", "falso" y "no sé".

Escala de creencias. La escala, con un índice de consistencia interna de 0.87 , constó de cinco reactivos orientados a evaluar las creencias positivas de los padres acerca de que sus hijos usen condón, cuyas opciones de respuesta se mostraron en una escala Likert con valores de 1 ("totalmente desacuerdo") a 5 ("totalmente de acuerdo"). 
Escala de estereotipos. Esta escala, con un coeficiente alfa de Cronbach de 0.85 , se conformó con ocho reactivos referentes a los estereotipos que prevalecen en torno a lo que está mal visto que hagan hombres y mujeres en el cuidado de su salud sexual. La opciones de respuesta se presentaron en una escala Likert con valores de 1 ("totalmente desacuerdo") a 5 ("totalmente de acuerdo").

Escala de expectativas hacia la comunicación sexual con los hijos. Dicha escala, con un coeficiente de alfa de 0.90 , estuvo integrada por siete reactivos referentes a lo que esperan los padres que ocurra si hablan con sus hijos sobre su sexualidad (por ejemplo, "Sería menos probable que mi hija se embarace"). Las opciones de respuesta se expusieron en una escala Likert con valores de 1 ("totalmente en desacuerdo") a 5 ("totalmente de acuerdo").

Escala de comunicación sobre temas sexuales. La escala de comunicación, con un índice de consistencia interna de 0.94 , se conformó por 17 reactivos referentes a tres tópicos: métodos anticonceptivos (seis reactivos), relaciones sexuales (seis reactivos) y VIH/sida (cinco reactivos). Las opciones de respuesta para cada reactivo se presentaron en una escala Likert, con valores de 1 ("nunca") a 5 ("una gran cantidad de veces").

\section{Procedimiento}

Con la anuencia de las autoridades escolares, se convocó a los padres a una reunión en la que un investigador entrenado les explicó los objetivos, el carácter confidencial y anónimo de sus respuestas y las instrucciones para el llenado de los instrumentos. El investigador estuvo presente durante el tiempo que demoraron los padres en responderlos (30 minutos en promedio), esto con el propósito de aclarar las dudas que surgieran durante el proceso. Las respuestas de los participantes se capturaron en una base de datos utilizando el programa SPSS para Mac, versión 20.

\section{RESULTADOS}

En la Tabla 1 se presentan los resultados de la comparación entre los padres de hijos con discapacidad
(PCD) y los padres de hijos sin discapacidad (PSD) respecto a las características sociodemográficas. No se encontraron diferencias estadísticamente significativas entre ambos grupos en términos de edad, sexo y estado civil. No obstante, el grupo PCD registró una mayor escolaridad que el PSD.

Tabla 1. Datos sociodemográficos.

\begin{tabular}{|l|c|c|c|}
\hline \multicolumn{1}{|c|}{ Características } & $\begin{array}{c}\text { PCD } \\
(\mathbf{n}=\mathbf{7 6})\end{array}$ & $\begin{array}{c}\text { PSD } \\
(\mathbf{n}=76)\end{array}$ & $\boldsymbol{p}$ \\
\hline Edad (años) & $45 \pm 8$ & $44 \pm 7$ & NS \\
\hline Sexo (F/M) & $58 / 18$ & $60 / 16$ & NS \\
\hline \multicolumn{5}{|l|}{ Estado civil } & 8 & 7 & NS \\
\hline Soltero & 46 & 45 & \\
\hline Casado & 10 & 11 & \\
\hline Unión libre & 4 & 1 & \\
\hline Divorciado & 5 & 7 & \\
\hline Separado & 3 & 5 & \\
\hline Viudo & & & .000 \\
\hline Escolaridad & 41 & 51 & \\
\hline Educación básica & 19 & 22 & \\
\hline Educación media & 14 & 3 & \\
\hline Educación superior & & &
\end{tabular}

Los resultados indican que los padres de ambos grupos mostraron un bajo nivel de conocimientos sobre ITS y VIH/sida, y más bajo aún en conocimientos sobre uso del condón. Ambos grupos señalaron tener actitudes positivas hacia el uso el condón por parte de sus hijos. En el caso de los estereotipos, el grupo PCD obtuvo una puntuación significativamente mayor respecto al PSD. En ambos grupos se registraron expectativas positivas altas hacia la comunicación sobre sexualidad; sin embargo, el PCD obtuvo puntuaciones más bajas que el PSD en cuanto a la comunicación sobre métodos anticonceptivos, relaciones sexuales y VIH/ sida (Tabla 2).

Posteriormente, se compararon los grupos PCD y PSD en cada uno de los reactivos que conforman la escala de comunicación sobre sexualidad. Los resultados de la prueba $t$ de Student indican diferencias significativas entre ambos grupos $(p<.05)$. Los PSD, en comparación con los PCD, hablaban más sobre cada uno de los temas. En el grupo de PCD los temas de los que más hablaban eran las formas de manejar la presión de amigos y pareja ante la posibilidad de tener relaciones sexua- 
Tabla 2. Comparación entre ambos grupos de padres.

\begin{tabular}{|c|c|c|c|c|c|c|}
\hline \multirow{2}{*}{ Variables } & \multicolumn{2}{|c|}{$\begin{array}{c}\text { PCD } \\
(n=76)\end{array}$} & \multicolumn{2}{|c|}{$\begin{array}{c}\text { PSD } \\
(n=76)\end{array}$} & \multirow[t]{2}{*}{ t } & \multirow{2}{*}{$p$} \\
\hline & M & D.E. & M & D.E. & & \\
\hline Conocimientos sobre ITS y VIH/sida & 61.61 & 15.74 & 62.94 & 14.48 & 0.477 & 634 \\
\hline Conocimientos sobre el uso del condón & 37.10 & 23.57 & 31.33 & 23.11 & -1.425 & .157 \\
\hline Creencias positivas & 3.99 & 1.01 & 4.23 & 0.94 & 1.44 & .150 \\
\hline Estereotipos hacia el cuidado de la salud & 2.02 & 0.89 & 1.86 & 0.67 & -2.191 & .030 \\
\hline Expectativas positivas & 3.86 & 0.76 & 3.91 & 1.02 & 0.358 & .721 \\
\hline $\begin{array}{l}\text { Comunicación sobre métodos anticon- } \\
\text { ceptivos }\end{array}$ & 1.85 & 0.94 & 2.89 & 1.04 & 5.695 & .000 \\
\hline Comunicación sobre relaciones sexuales & 2.32 & 1.13 & 3.33 & 1.03 & 4.909 & .000 \\
\hline Comunicación sobre VIH/sida & 2.13 & 1.00 & 3.35 & 0.91 & 6.928 & .000 \\
\hline
\end{tabular}

les, el negarse a una propuesta sexual y las posibles consecuencias de un embarazo; por el contrario, de los que menos hablaban fueron los referidos de las formas de usar un condón, la forma de los condones masculinos y los establecimientos en los que pueden adquirirse condones. En el caso de los PSD, los temas que platicaban con sus hijos con mayor frecuencia eran cómo protegerse en las relaciones sexuales, cómo prevenir las ITS y el VIH/sida y las posibles consecuencias de un embarazo; los temas menos abordados fueron semejantes a los PCD. En la Figura 1 se muestran tales resultados.

Figura 1. Comunicación sexual entre grupos.

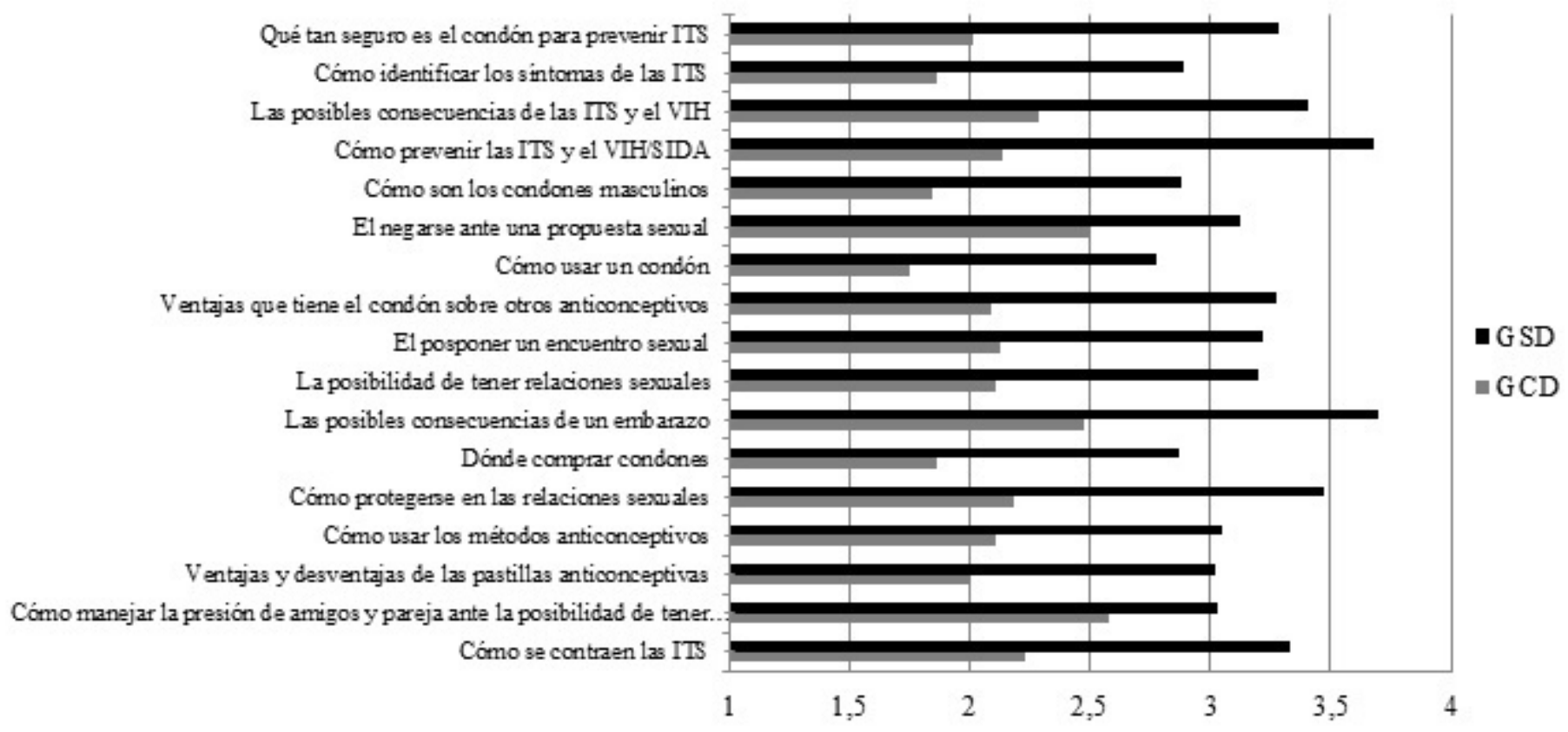

Por último, se hizo un análisis comparativo para conocer el papel que desempeñan las características de los padres en la comunicación sobre sexualidad con sus hijos. Los resultados indican que en

ambos grupos, los hombres, en comparación con las mujeres, conversaban más sobre dichas cuestiones (Tabla 3). 
Tabla 3. Análisis comparativo de la comunicación sobre sexualidad por sexo.

\begin{tabular}{|l|c|c|c|c|c|c|c|c|}
\hline \multirow{2}{*}{ Variable } & \multicolumn{6}{|c|}{ Comunicación sobre sexualidad } \\
\cline { 2 - 9 } & \multicolumn{4}{|c|}{$\begin{array}{c}\text { PCD } \\
(\mathbf{n = 7 6})\end{array}$} & \multicolumn{5}{c|}{$\begin{array}{c}\text { PSD } \\
(\mathbf{n}=76)\end{array}$} \\
\hline Sexo & Media & D.E. & t & $\boldsymbol{p}$ & Media & D.E. & t & $\boldsymbol{p}$ \\
\hline Hombres & 2.16 & 0.892 & .440 & .662 & 3.34 & 0.957 & 0.609 & .546 \\
\hline Mujeres & 2.04 & 0.99 & & & 3.11 & 0.944 & & \\
\hline
\end{tabular}

Respecto a la edad de los padres, en el grupo de PCD los de mayor edad conversaban con más frecuencia con sus hijos, lo opuesto a los PSD, entre quienes eran los padres más jóvenes quienes dialogaban más frecuentemente con sus hijos. Los padres divorciados en ambos grupos platicaban con mayor frecuencia sobre los temas ya señalados. No obstante, estas diferencias no fueron estadísticamente significativas; únicamente resultó serlo la escolaridad, y exclusivamente en los PCD, lo que indica que en ese grupo los padres con educación superior dialogaban con sus hijos sobre la sexualidad con mayor frecuencia, a diferencia de quienes tenían solamente educación básica (Tabla 4).

Tabla 4. Análisis comparativo de la comunicación sobre sexualidad por edad, estado civil y escolaridad.

\begin{tabular}{|l|c|c|c|c|c|c|c|c|}
\hline \multirow{2}{*}{ Variable } & \multicolumn{6}{|c|}{ Comunicación sobre sexualidad } \\
\cline { 2 - 10 } & \multicolumn{5}{|c|}{$\begin{array}{c}\text { PCD } \\
\text { (n= 76) }\end{array}$} & \multicolumn{5}{c|}{ PSD } \\
(n= 76) \\
\hline Edad & M & D.E. & F & p & M & D.E. & F & $p$ \\
\hline Menores de 39 años & 1.99 & 975 & 0.808 & .450 & 3.42 & 0.67 & 0.62 & .543 \\
\hline De 40 a 50 años & 1.98 & 1.04 & & & 3.15 & 1.11 & & \\
\hline Mayores a 51 años & 2.31 & 0.77 & & & 2.96 & 0.79 & & \\
\hline Estado civil & & & & & & & & \\
\hline Soltero & 2.09 & 1.06 & 1.060 & .390 & 3.41 & 0.79 & 1.81 & .135 \\
\hline Casado & 2.11 & 0.93 & & & 3.25 & 0.98 & & \\
\hline Unión libre & 1.55 & 0.89 & & & 2.43 & 0.67 & & \\
\hline Divorciado & 2.88 & 1.66 & & & 3.78 & 0.75 & & \\
\hline Separado & 2.15 & 0.56 & & & 3.61 & 0.62 & & \\
\hline Viudo & 2.22 & 1.06 & & & 2.12 & 1.35 & & \\
\hline Escolaridad & & & & & & & & \\
\hline Básica & 1.83 & 0.92 & 4.470 & .015 & 3.04 & 0.91 & 1.13 & .333 \\
\hline Media superior & 2.27 & 0.83 & & & 3.48 & 1.03 & & \\
\hline Superior & 2.66 & 0.99 & & & 2.74 & 0.24 & & \\
\hline
\end{tabular}

\section{DISCUSIÓN}

El objetivo central de esta investigación fue comparar las características sociodemográficas, conocimientos, creencias, estereotipos, expectativas y comunicación sobre sexualidad entre padres de hijos con y sin discapacidad. Los resultados más importantes señalan que hubo entre ellos diferencias significativas, las cuales se discuten a continuación.
En primer lugar, a partir de los resultados obtenidos por los padres en las pruebas de conocimiento fue posible identificar que tanto los PCD como los PSD carecen de información relevante sobre las formas de transmisión y prevención del ITS, VIH y embarazos no deseados, y desconocen aun más las medidas que se deben tomar para asegurar el uso correcto del preservativo. Es posible que en la época en que ellos tuvieron su primera relación sexual todavía no hubiera suficiente difusión sobre la epidemia del VIH ni información acerca de su prevención mediante el uso del condón. 
Aunque en diferentes estudios se ha desdeñado el papel que desempañan los conocimientos, estos son una herramienta fundamental ya que permiten que los padres transmitan a sus hijos información útil y certera sobre estos problemas.

Por otra parte, se encontró que los padres de ambos grupos expresaron tener ideas poco estereotipadas (menores aún en los PSD), evaluar positivamente el uso de condón por parte de sus hijos y considerar que hablar con ellos sobre temas de sexualidad puede evitar que se embaracen o se contagien con ITS. Sin embargo, en los hechos, los PCD hablaban menos sobre dichos problemas que los PSD.

Algo que resulta interesante es el análisis de los temas que se discutían en cada grupo. En los PCD, los tópicos que se abordaban más se orientaban sobre todo a evitar que sus hijos tuvieran relaciones sexuales (por ejemplo: cómo manejar la presión de los amigos y la pareja ante la posibilidad de tener relaciones sexuales, el negarse a una propuesta sexual y las posibles consecuencias de un embarazo); los temas menos tratados tienen que ver con el cuidado de su salud sexual mediante el uso del preservativo (cómo usar un condón, cómo son los condones masculinos y dónde comprarlos). Tales resultados sugieren que los padres que tienen hijos con discapacidad están más preocupados de que sus hijos tengan relaciones sexuales que en su cuidado sexual. Una posible explicación puede ser que, ante ese temor, optan por evadir el tema, tal como señalan algunos autores (López, 2006; Morales et al., 2011).

Por su parte, los PSD conversan con mayor frecuencia con sus hijos sobre temas que pueden reflejar su aprensión de que sus hijos adquieran ITS o se embaracen (por ejemplo: cómo protegerse en las relaciones sexuales, cómo prevenir las ITS y el VIH/sida y las posibles consecuencias de un embarazo). Sin embargo, al igual que los PCD, no brindan información precisa que facilite a sus hijos saber dónde adquirir y cómo usar preservativos.
Respecto a las características de los padres, en otras investigaciones se ha señalado que el sexo (Meshke et al., 2002 ), la edad (Einsenberg et al., 2005) y el nivel educativo (Gallegos et al., 2007) desempeñan un papel relevante en la comunicación sobre sexualidad que los padres establecen con sus hijos; no obstante, en el presente estudio fue únicamente la escolaridad la que favoreció dicha comunicación, particularmente en los PCD. Para comprender este resultado, es importante recordar que en el grupo de PCD se concentró una mayor cantidad de padres con educación superior que en el de PSD. Ahora bien, la investigación demuestra que cuando los padres alcanzan un nivel educativo mayor, aumentan parejamente sus conocimientos sobre diversos aspectos relativos a la sexualidad (Bárcena et al., 2013) y, consecuentemente, conversan más con sus hijos a ese respecto. Es posible suponer entonces que cuando los padres están más informados, comparten más fácilmente esos datos con sus hijos a través del diálogo. Por el contrario, cuando los padres tienen un nivel educativo bajo manejan deficientemente la información sobre sexualidad, lo que dificulta su comunicación.

Finalmente, los resultados más importantes del estudio presente destacan la importancia de proveer a los padres de los conocimientos necesarios. En el caso de los PCD, es preciso fomentar en ellos habilidades comunicativas que les hagan posible hablar con sus hijos sobre estos tópicos. En suma, estos resultados apoyan el llamado global para una mayor defensa de las personas con discapacidad y de sus familias ante la experiencia de la sexualidad. La comunicación sobre la sexualidad que los padres establezcan con sus hijos podría reducir la violencia, la discriminación y las enfermedades que pueden contraer por mera ignorancia, mismas que afectan tanto a las propias personas con discapacidad como a sus familias (Alianza Internacional de la Discapacidad, y Centro de Derechos Reproductivos, 2015).

\section{REFERENCIAS}

Alianza Internacional de la Discapacidad y Centro de Derechos Reproductivos (2015). Educación sexual integral e inclusiva y la CDPD: Sumisión a Medio Día de Debate General del Comité de la CDPD sobre el Derecho a la Educación, Palacio de las Naciones, Ginebra. Disponible en línea: http://www.ohchr.org/EN/HRBodies/CRPD/Pages/DGDontherighttoeducationforpersonswithdisabilities.aspx. 
Ballan, M.S. (2012). Parental perspectives of communication about sexuality in families of children with autism spectrum disorders. Journal of Autism and Developmental Disorders, 42(5), 676-684.

Bárcena, S. (2013). Efectos diferenciales de un programa de VIH dirigido independientemente a padres e hijos. Tesis doctoral inédita. México: UNAM.

Bárcena, S., Robles, S. y Díaz-Loving, R. (2013). El papel de los padres en la conducta sexual de sus hijos. Acta de Investigación Psicológica, 3(1), 956-968.

Caricote, E. (2008). Influencia de los padres en la educación sexual de los adolescentes. Investigación Arbitrada, 12(40), 79-87.

Centro Nacional para la Prevención y el Control del VIH/sida (CENSIDA) (2014). Casos acumulados de sida por grupos de edad y sexo. Disponible en línea: http://www.censida.salud.gob.mx/descargas/2010/15nov2010/casos_grupoedadysexo.pdf.

Cuskelly, M. y Bryde, R. (2004). Attitudes towards the sexuality of adults with an intellectual disability: Parents, support staff, and a community sample. Journal of Intellectual and Developmental Disability, 29, 255-264.

DiIorio, C., Pluhar, E. y Belcher, L. (2003). Parent-child communication about sexuality: A review of the literature from 19802002. Journal of HIV/AIDS Prevention and Education for Adolescents and Children, 5, 7-31.

Eisenberg, M.E., Sieving, C., Bearinger, L.H., Swain, C. y Resnick, M.D. (2005). Parental notification laws for minors' access to contraception - What do parents say? Archives of Pediatritcs \& Adolescent Medicine, 159, 120-125.

Frías, B., Barroso, R., Rodríguez, M., Moreno, D. y Robles, S. (2008). Comunicación sobre temas sexuales con padres y pareja y su relación con la intención para tener sexo y usar condón en adolescentes. En: S. Rivera Aragón, R. Díaz-Loving, R. Sánchez Aragón e I. Reyes Lagunes (Eds.), La Psicología Social en México (pp. 227-232), Vol. XII, México, D.F.: AMEPSO.

Gallegos, E.C., Villaruel, M.A, Gómez, M., Onofre, D.J. y Zhou, Y. (2007). Research brief: Sexual communication and knowledge among mexican parents and their adolescent children. Journal of the Association of Nurses in AIDS Care, 18(2), 28-34.

Gutiérrez, B. (2010). Habilidades sociosexuales en personas con discapacidad intelectual. España: Pirámide.

Harrell, E. (2015). Crime againts persons with disabilities, 2009-2013-Statical Tables. Bureau of Justice Statics, 1-22.

Instituto Nacional de Estadística y Geografía (2010). Porcentaje de nacimientos registrados de madres adolescentes (menores de 20 años) 2008-2010. Disponible en línea: http://www.inegi.org.mx/lib/olap/general/MDXQueryDatos.asp.

Instituto Nacional de Estadística y Geografía y Consejo Nacional de Población (2015). Encuesta Nacional de Dinámica Demográfica. México: INEGI/CONAPO.

Jerman, P. y Constantine, N.A. (2010). Demographic and psychological predictors of parent- adolescent communication about sex: A representative statewide analysis. Journal of Youth and Adolescence, 39, 1164-1174.

Karellou, J. (2007). Parents' attitudes towards the sexuality of people with learning disabilities in Greece. Journal on Developmental Disabilities, 13, 55-72.

Lefkowitz, E.S. y Stoppa, T.M (2006). Positive sexual communication and socialization in the parent-adolescent context. New Directions for Child and Adolescent Development, 112, 39-55.

Lehr, S.T., Demi, A.S., Dilorio, C. y Facteau, J. (2005). Predictors of fathers-son communication about sexuality. The Journal of Sex Research, 42(2), 119-129.

López, F. (2006). La educación sexual. Madrid: Biblioteca Nueva.

Markham, C.M., Lormand, D. y Gloppen, K.M. (2010). Connectedness as a predictor of sexual and reproductive health outcomes for youth. Journal of Adolescent Health, 46, S23-S24.

Meshke, L.L., Bartolomé, S. y Zentael, S.R. (2002). Adolescent sexuality and parent-adolescent processes: Promoting healthy teen choices. Journal of Adolescent Health, 31, 264-269.

Morales G., E., López E., O. y Mullet, E. (2011). Acceptability of sexual relationships among people with learning disabilities: Family and professional caregivers' views in Mexico. Sexuality \& Disability, 29, 165-174.

Navarro N., Z. y Hernández A., I. (2012). Influencia de las actitudes de los padres ante la educación sexual y la discapacidad intelectual. Psicología y Salud, 22(2), 195-203.

Organización Mundial de la Salud (2015). Discapacidad y salud. Disponible en línea: http://www.who.int/mediacentre/factsheets/fs352/es/.

Organización Mundial de la Salud (2016). Encuesta Mundial de Salud a Escolares. Disponible en línea: http://www.who.int/chp/ gshs/es/.

Pownall, J.D., Jahoda, A., Hastings, R. y Kerr, L. (2011). Sexual understanding and development of young people with intellectual disabilities: Mothers' perspectives. American Journal of Intellectual and Developmental Disabilities, 116, 205-219.

Rivera, P. (2008). Sexualidad de los niños, niñas y jóvenes con discapacidad. Revista Educación, 32(1), 157-170.

Robles, S., Guevara, Y., Pérez, Y. y Hermosillo, A. (2013). Comunicación con padres y conducta sexual en jóvenes mexicanos con discapacidad auditiva. Psicología y Salud, 23(2), 227-239.

Schaalma, H.P., Abraham, C., Gillmore, M.R. y Kok, G. (2004). Sex education as health promotion: "What does it take?". Archives of Sexual Behavior, 33(3), 259-269. 
Secretaría de Educación Pública (2016). Sistema interactivo de consulta de estadística educativa. Disponible en línea: http:// www.planeacion.sep.gob.mx/principalescifras/.

Shearer, C.L., Hosterman, S.J., Gillen, M.M. y Lefkowitz, E.S. (2005). Are traditional gender role associated with risky sexual behavior and condom-related beliefs? Sex Roles, 52(5), 311-324.

World Bank (2009). A population at risk: HIV/AIDS and disability: Capturing hidden voices. Findings from the Yale/World Bank Global Survey of HIV/AIDS and Disability. Washington D.C.: World Bank. 